\title{
Roles of unphosphorylated STATs in signaling
}

\author{
Jinbo Yang ${ }^{1,2}$, George R Stark ${ }^{2}$ \\ ${ }^{I}$ School of Biological Science, Lanzhou University, Lanzhou, Gansu 730000, China; ${ }^{2}$ Department of Molecular Genetics, Lerner \\ Research Institute, The Cleveland Clinic, Cleveland, OH 44195, USA
}

The seven members of the signal transducer and activator of transcription (STAT) family of transcription factors are activated in response to many different cytokines and growth factors by phosphorylation of specific tyrosine residues. The STAT1 and STAT3 genes are specific targets of activated STATs 1 and 3, respectively, resulting in large increases in the levels of these unphosphorylated STATs (U-STATs) in response to the interferons (STAT1) or ligands that active gp130, such as IL-6 (STAT3). U-STATs drive gene expression by novel mechanisms distinct from those used by phosphorylated STAT (P-STAT) dimers. In this review, we discuss the roles of U-STATs in transcription and regulation of gene expression.

Keywords: transcription, cytokines, interferons, IL-6

Cell Research (2008) 18: 443-451. doi: 10.1038/cr.2008.41; published online 25 March 2008

\section{Introduction}

Signal transducers and activators of transcription (STATs) were originally discovered as DNA-binding proteins that mediate interferon (IFN)-dependent gene expression [1-3]. STATs are latent in the cytoplasm until they are activated by extracellular ligands, including cytokines, growth factors, and hormones $[4,5]$. Binding of these extracellular ligands to their specific receptors leads to the activation of various tyrosine kinases (TKs), including JAKs, receptor TKs, and non-receptor TKs such as SRC and ABL, which can directly phosphorylate STATs in the absence of ligand-induced receptor signaling $[6,7]$. These TKs phosphorylate a single tyrosine residue of each STAT, followed by homo- or hetero-dimerization of STATs through reciprocal Src homology 2 (SH2)-phosphotyrosine interactions, leading to nuclear translocation of the resulting dimers and activation of target genes. Seven STATs have been identified in mammals: STAT1, STAT2, STAT3, STAT4, STAT5A, STAT5B, and STAT6. Each is differentially activated by specific extracellular ligands, allowing differential intracellular processing of signals transduced across the plasma membrane. The biological role of each STAT has been delineated by investigating the phenotypes

Correspondence: George R Stark ${ }^{\mathrm{a}}$, Jinbo Yang ${ }^{\mathrm{b}}$

Tel: +1-216-444-6062; Fax: +1-216-444-0512

aE-mail: starkg@ccf.org

bE-mail: yangj@ccf.org of mice lacking their expression [5]. Recently, it has been found that STATs 1 and 3 (and probably other STATs) also play important roles in mediating gene expression without tyrosine phosphorylation. The expression of unphosphorylated STATs (U-STATs) 1 and 3 is greatly increased in response to their activation, since the STAT1 gene is strongly activated by phosphorylated STAT (P-STAT) 1 dimers or ISGF3, which are formed in response to type II or type I IFNs, respectively, and, similarly, since the STAT3 gene is strongly activated by P-STAT3 dimers, which are formed in response to IL- 6 and other ligands that activate the gp130 common receptor subunit. Ligand-dependent increases in the concentrations of U-STATs drive the expression of genes that are distinct from those activated by P-STATs. The abnormally high levels of U-STAT3 that accompany the abnormal constitutive activation of STAT3 found in many tumors drive the over-expression of several genes that contribute to tumorigenesis.

U-STAT1 mediates constitutive expression of the low molecular mass polypeptide (LMP) 2 gene by collaborating with IRF1 [8], and U-STAT3 binds to unphosphorylated $\mathrm{NF} \kappa \mathrm{B}(\mathrm{U}-\mathrm{NF} \kappa \mathrm{B})$, in competition with I $\mathrm{B}$, and the resulting U-STAT3/U-NFאB complex accumulates in the nucleus with help from the nuclear localization signal of STAT3, activating a subset of $\kappa \mathrm{B}$-dependent genes (Figure 1) [9]; U-STAT6 cooperates with $\mathrm{p} 300$ and binds to a consensus STAT6 binding site located within the COX-2 promoter to enhance COX-2 expression [10]. The discovery of these specific mechanisms for how U-STATs mediate gene expression serves as examples for additional mechanisms 


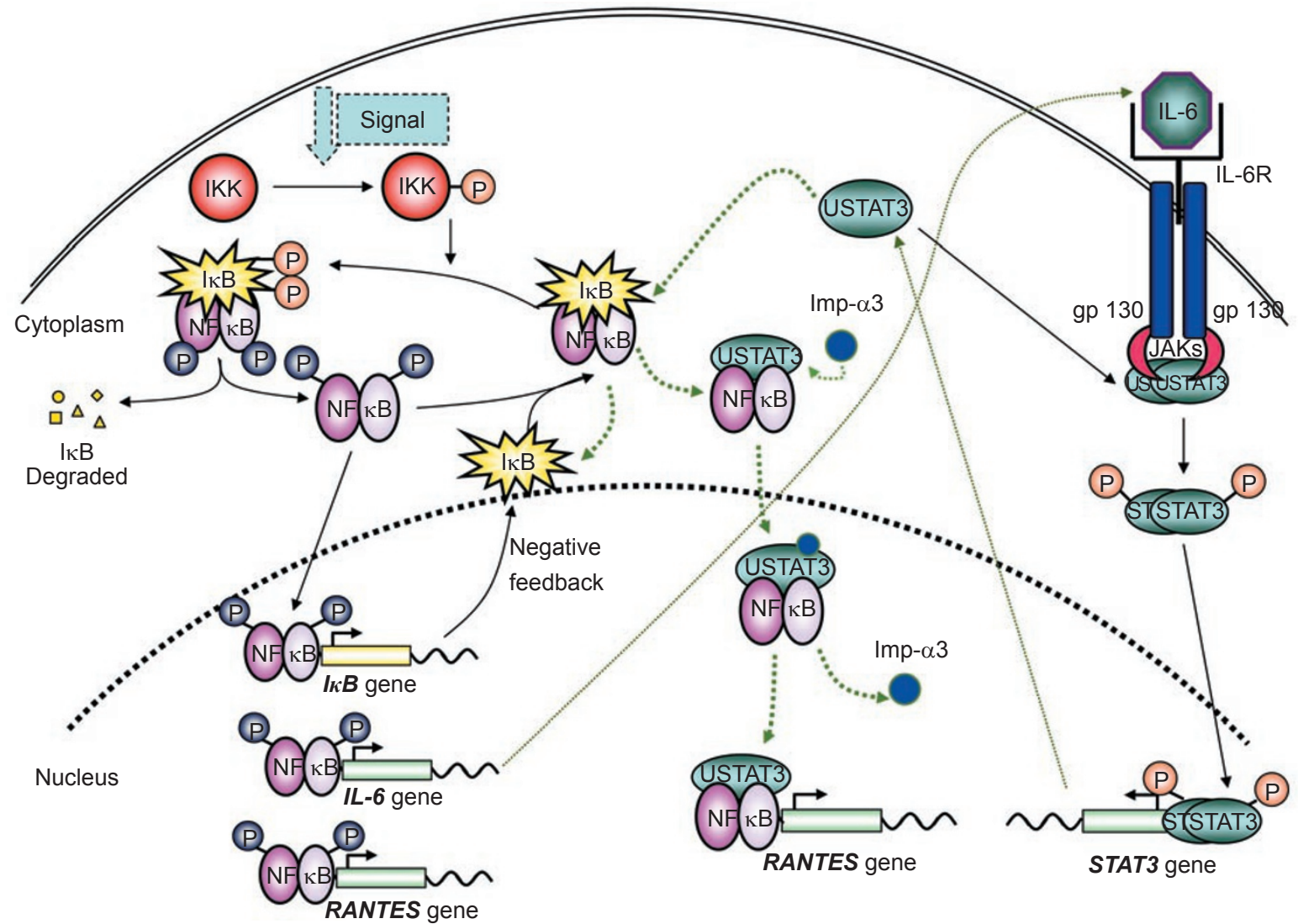

Figure 1 Interactions between the STAT3 and NFKB pathways. U-STAT3, induced to a high level due to activation of the STAT3 gene in response to ligands such as IL-6, competes with $I_{\kappa} B$ for p65/p50. The U-STAT3:U-NF $\kappa B$ complex activates the RANTES promoter plus a subset of other promoters that have $\kappa B$ elements. U-STAT3 also drives the expression of some genes that do not have $\kappa B$ elements, by an unknown mechanism. The $\kappa B$ element of the IL- 6 gene is driven by canonical NF $\kappa B$ signaling in response to ligands such as TNF- $\alpha$ or IL-1, setting up a positive feedback loop that is driven by the activation of STAT3 in response to secreted IL-6, leading to an increased level of U-STAT3 that sustains the activation of genes such as RANTES.

that have yet to be revealed. Many of the STATs are sticky and interact with many different proteins. STAT1 and IRF1 bind to each other even in the absence of DNA, and this heterodimer binds to a composite element in the LMP2 promoter that recognizes each monomeric component separately. The ternary complex is stable enough to drive the constitutive expression of LMP2, but can be displaced by the more potent STAT1 dimer in IFN $\gamma$-treated cells. STATs can form several different homo- and heterodimers and STATs can form heterodimers with other proteins even without tyrosine phosphorylation. Different complexes, which may not be very stable as free forms in solution, can nevertheless form on specific target genes, depending on the stabilization through binding to specific DNA elements in each promoter and probably also through specific interactions with other bound proteins. Thus, we expect many different mechanisms for U-STAT-dependent expression of different genes.

\section{STAT1-dependent gene expression}

STAT1, the first member of the multi-gene family to be discovered, is a principal target of both type I and type II IFNs [2, 11-13]. STAT1-null (STAT1 ${ }^{--}$) mice have lost responsiveness to both types of IFN and have thus acquired enhanced susceptibility to bacterial and viral pathogens [14]. The discovery that the active form of STAT1 is required for IFN- $\alpha$ and IFN- $\gamma$ to inhibit the growth of cultured cells [15-17] has led to the assumption that inadequate function of STAT1 might result in cell growth deregulation and disturbed immune function, i.e. disorders that are pertinent to malignancy. The potential involvement of STAT1 in cancer has been supported by several observations reporting inappropriate activation of STAT1 and even loss of its expression in malignant cells derived from different histological types of tumors such as breast cancer, head and neck cancer, melanoma, leukemia, and lymphoma $[6,18$ - 
21]. However, convincing evidence on the role of STAT1 in malignant tumors was provided by studies employing $\mathrm{STAT}^{-/}$mice. Although some recent data supported that $\mathrm{STAT}^{-/-}$mice (therefore unresponsive to IFNs) lacking host-cell sensitivity to IFN $\gamma$ in antitumor immunity form more spontaneous and chemically induced tumors than wild-type mice [22], most literatures observed no increased spontaneous malignancy in STAT1 $1^{-/}$mice. They manifested heightened susceptibility to both chemically induced and transplanted tumors compared to control animals [23, 24]. These data support the idea that STAT1 might function as a tumor suppressor [25]. Subsequent studies revealed that the involvement of STAT1 in oncogenesis is more complex and that only a part of its tumor growth-suppressing activity is attributable to the loss of IFN-dependent anti-proliferative response when STAT1 is absent. Kaplan et al. [23] demonstrated that the elevated growth of tumor cells in STAT1-deficient, IFN- $\gamma$ insensitive mice is at least partly due to the absence of well-known effects of IFN- $\gamma$ on tumor cell immunogenicity and/or host response to tumor antigens. Since IFN- $\gamma$ represents a critical component of the immune system required for tumor surveillance, it is likely that impaired STAT1 function negatively affects the immunogenic phenotype of developing tumors. Thus, the impaired responsiveness to IFN- $\gamma$ due to STAT1 dysfunction may provide a selective growth advantage to some malignant cells at an early stage of tumor development, a process known as cancer immuno-editing [26, 27]. Several reports showing that some spontaneous human tumors are selectively unresponsive to IFN- $\gamma$ due to perturbed STAT1 activation suggest that, similarly to animal models, STAT1dependent tumor surveillance also operates in humans [2832]. More recently, it was shown that STAT1 can accelerate the development of hematopoietic tumors independently of IFN-dependent signaling. These authors demonstrated that the up-regulation of MHC class I expression represents a general mechanism for escape from tumor surveillance and that a low level of MHC class I expression might be beneficial for leukemia patients [33].

The first indication that STAT1 might function as a transcription factor in the absence of tyrosine phosphorylation came from an analysis of its role in TNF-induced apoptosis [34]. STAT1-null U3A cells are resistant to TNF plus cycloheximide, while parental 2fTGH cells or STAT1deficient U3A cells reconstituted with wild-type STAT1 (U3A-R cells) are sensitive. Furthermore, the expression of caspases 1, 2, and 3 is defective in U3A cells and is induced only in $2 \mathrm{fTGH}$ and U3A-R cells but not in U3A cells following TNF treatment. Thus, STAT1 is required for TNF-mediated apoptosis and appears to act in the absence of any phosphorylation of tyrosine 701. Small amounts of constitutively P-STAT1 in 2fTGH and U3A-R cells are not responsible for the observed response since U3A-701 cells, reconstituted with the Y701F mutant of STAT1, which cannot be phosphorylated on tyrosine, are also sensitive to TNF-mediated apoptosis and normal for caspase gene expression [34].

Another indication of a role in transcription for STAT1 without tyrosine phosphorylation came from an analysis of the expression of a gene encoding a component of the 20S proteosome, LMP2 [8]. The bi-directional promoter that regulates LMP2 expression contains overlapping IFN consensus sequence 2 (ICS2) and gamma-activated sequence (GAS) sites. The IFN-inducible LMP2 gene is also transcribed at a lower level in the absence of IFN [35]. In vivo footprinting of the ICS2/GAS element revealed protein-DNA contacts at both sub-sites in unstimulated HeLa cells [36]. Thus, both STAT1 and IRF1 appear to be essential for basal transcription of the LMP2 gene. There is barely any LMP2 expression in IRF1-null mice [36]. STAT1-deficient U3A cells do not express LMP2, but the gene is transcribed in parental 2fTGH cells and its expression is restored when wild-type STAT1 is put back into U3A cells [8]. LMP2 transcription is also restored in U3A-701 cells, in which the mutant Y701F STAT1 cannot be phosphorylated [37].

Using DNA microarrays, a comparison of transcription in U3A cells and U3A-701 cells revealed that the basal expression of many genes is regulated similarly by wildtype U-STAT1 and STAT1 Y701F. The genes include those encoding the MHC class II transactivator (CIITA), hsp70, and Bcl-xL. MHC class I and 2-microglobulin expression was similar in U3A-701 and 2fTGH cells [37]. The expression of several caspase genes is also regulated similarly in U3A-701 and 2fTGH cells [34]. Constitutive expression of the MHC class I and $\beta 2$-microglobulin genes is lower in mouse STAT1-null T lymphocytes than in wild-type cells [38]. Several other examples of ligand-independent functions of STAT1 have been described more recently. In cardiac myocytes, transcriptional activation of Fas and FasL is dependent on S727 of STAT-1 but independent of Y701. Furthermore, S727 but not Y701 is required for the enhancement of cardiomyocyte cell death by STAT1 following ischemia/reperfusion injury [39]. In another instance, 7-ketocholesterol-induced apoptosis requires STAT1 since this phenomenon is not observed in U3A cells, but apoptosis is restored when either Y701F or wild-type STAT1 is put back into U3A cells. However, U3A cells reconstituted with S727A STAT1 are not sensitive to 7ketocholesterol-induced apoptosis [40].

Thus, serine 727 of STAT 1 appears to play an important role in some constitutive functions. Such a role is also indicated by the fact that U3A-S727A cells are resistant to TNFmediated apoptosis and do not express caspases 1,2, and 
3 [34]. Serine phosphorylation of STAT1 can be induced independently of tyrosine phosphorylation [41] and STAT1 can be phosphorylated only on serine 727 in response to UV [21], IL-1, or TNF [42]. The effect of the S727A mutation of STAT1 on basal transcription of caspase [34], GBP1, TAP1, and IFP53 genes [21] shows that this serine residue, which lies in the transactivation domain, is essential for STAT1 to be an effective transcription factor for certain genes. More examples were found recently. Timofeeva et al. [32] showed that, in Wilms' tumor (WT), one of the most common pediatric solid cancers, STAT1 was found to be constitutively phosphorylated on serine 727 in 19 of 21 primary WT samples and two WT cell lines. The inactivating mutation S727A reduced colony formation of WT cells in soft agar by more than $80 \%$ and induced apoptosis under conditions of growth stress. S727-P-STAT1 provided resistance to apoptosis for WT cells by upregulating the expression of heat-shock protein (HSP) 27 and the antiapoptotic protein myeloid cell leukemia (MCL)-1. These findings suggest that serine-P-STAT1 plays a critical role in the pathogenesis of WT and other neoplasms. Although it is tempting to suggest that the phosphorylation of S727 is important for this function, there is at present no direct evidence to rule out the possibility that $\mathrm{S} 727$ is important per se, without phosphorylation, for vital protein-protein interactions on certain promoters.

The gene expression patterns induced by U-STAT1 depend strongly on the cell type. Gene expression profiles performed in human fibroblast BJ cells and epithelial HME1 cells in which Y701F-STAT1 was stably expressed at a high level show quite different patterns of gene expression (H Cheon and GR Stark, unpublished data).

\section{STAT3-dependent gene expression}

STAT1 has been shown to drive gene expression in the absence of tyrosine phosphorylation [43]. In the case of STAT3, an array-based analysis of gene expression revealed that the relative levels of more than a thousand mRNAs changed in response to increased expression of wild type or Y705F-STAT3. In human primary epithelial hTERT-HME1 cells, 1420 mRNAs changed, positively or negatively, by more than 2-fold in response to a high level of wild-type STAT3 and 1191 mRNAs changed by the same amount in response to a high level of Y705F-STAT3. Furthermore, 869 mRNAs changed in common by at least 2 -fold in both cell populations, and 84 of these changed by more than 4fold [44]. These mRNAs include several that are induced to very high levels (10- to 40-fold). Analysis of mouse cell lines provided complementary data. In addition to the obvious cell-type differences in the two experiments, mouse cells with high level of wild-type or Y705F-STAT3 are compared with STAT3-null cells. In MEFs, more than a thousand mRNAs changed by more than 2-fold in cells with either high level of wild-type STAT3 or high level of Y705F-STAT3. Furthermore, about 400 mRNAs changed in common by more than 2-fold in both cell types [44]. Some of these genes are known to be regulated by STAT3 homodimers, including SOCS-3 [45], c-Myc and DP1 [46], c-Fos and c-Jun [47-49], and Bcl-x [50].

STAT3 was initially identified as an IL-6-dependent transcription factor important for mediating the inflammatory response, tumorigenesis, and anti-apoptotic effects on cells. Recently, in addition to its ability to form complexes with itself and other STATs, there are several reports that STAT3 and NFאB interact with each other [51]. For example, Hagihara et al. [52] demonstrated that STAT3 forms a complex with the p65 subunit of NFKB following stimulation of cells with IL-1 plus IL- 6 , and that the bound STAT3 interacts with non-consensus sequences near the $\kappa \mathrm{B}$ element of the serum amyloid A (SAA) promoter. Moreover, the complex formed by STAT3, p65, and p300 is essential for the synergistic induction by IL-1 and IL-6 of the SAA gene, which does not have a typical STAT3 response element in its promoter. Yu et al. [53] found that U-STAT3, through direct interaction with p65, serves as a dominant-negative inhibitor to suppress the ability of P$\mathrm{NF} \kappa \mathrm{B}$ to induce cytokine-dependent activation of the iNOS promoter in mesangial cells. Yoshida et al. [54] showed that STAT3 and $\mathrm{p} 65$ physically interact in vivo and that $\mathrm{p} 65$ homodimers can cooperate with U-STAT3 when bound to a specific type of $\kappa \mathrm{B}$ motif. Reciprocally, this interaction appears to inhibit the function of STAT3 GAS-binding sites. In contrast, the $\mathrm{p} 50$ subunit of $\mathrm{NF} \kappa \mathrm{B}$ can cooperate with P-STAT3 bound to GAS sites [54]. Yang et al. [9, 44] demonstrated that an increase in the concentration of endogenous U-STAT3 following long-term treatment with IL-6 allows U-STAT3 to compete effectively with IкB for $\mathrm{U}-\mathrm{NF} \kappa \mathrm{B}$, to form a novel transcription factor that induces RANTES expression by binding to the proximal $\kappa \mathrm{B}$ site of the RANTES promoter. Since the Y705F mutant of STAT3, which cannot be phosphorylated on tyrosine, also activates RANTES expression, this function of U-STAT3 is clearly distinct from the absolute requirement for tyrosine phosphorylation that enables STAT3 dimers to bind to GAS sequences [55-57].

It should be noted that several researchers have reported that additional phosphorylation of P-STAT3 dimers on Ser727 is needed for maximal activation of transcription, but not for DNA binding $[4,55]$. However, Ser 727 phosphorylation seemed to be not required for activation of RANTES, because STAT3- $\beta$, a truncate form of STAT3 which lacks 50 amino acids from the $\mathrm{C}$-terminus, is still capable of driving RANTES expression. It seems possible 
that the C-terminal domain, with Ser727 phosphorylated, might facilitate the transactivation of promoters other than RANTES in response to binding of the U-STAT3:UNFkB complex. For example, Ng et al. [58] have shown that STAT3 is phosphorylated on Ser727 but not Tyr705 in response to activation of the TrkA receptor by nerve growth factor, and that serine-P-STAT3 is important in driving signal-dependent gene expression.

Another recent example is that U-STAT6 forms a complex with p300, which is able to directly bind to the cyclooxygenase-2 promoter [10]. Surprisingly, the USTAT6/p300 complex binds to the same GAS element that is presumed to be bound by P-STAT homo- or heterodimers. This result is different from the data obtained by Yang et al. [9], Yoshida et al. [54] and Chatterjee-Kishore et al. [37] for U-STAT3 and U-STAT1.

\section{Nuclear-cytoplasmic transport of U-STATs}

Although all STATs share sequence and domain structures, each individual STAT protein that has been studied has evolved a distinct mechanism to regulate its intracellular trafficking. Specifically, the movement of STAT1, STAT2, and STAT3 into the nucleus is governed by different kinds of importin transporters. STAT family members have similar nuclear-localization signals (NLSs) or nuclearexport signals (NESs) domains that govern their passage into and out of the nucleus. STAT1 can be detected in the nuclei of $2 \mathrm{fTGH}$ cells in the absence of ligand-dependent stimulation, as can Y701F STAT1 [43]. A survey of several established cell lines shows that STAT1 is present in the nuclei of all cells in the absence of ligand-dependent stimulation. Treating cells with IFN $\gamma$ induces an increase in nuclear STAT1 and, although staurosporine inhibits the IFN $\gamma$-induced import of STAT1 into nuclei, it does not alter basal levels of nuclear STAT1 [59]. Unlike the nuclear import of tyrosine-P-STAT1, that of U-STAT1 is sensitive to wheat germ agglutinin and occurs independently of the import receptor p97 [59]. Soon after the discovery of the importin- $\alpha /$ importin- $\beta$-dependent nuclear import pathway, importin- $\alpha 5$ was found to mediate nuclear translocation of tyrosine-P-STAT1 $[60,61]$. Importin- $\alpha 5$ recognizes tyrosine-P-STAT1 either in the form of ISGF3 or as a homodimer [62]. In this case, tyrosine phosphorylation of STAT1 induces conformational changes that promote STAT/STAT interactions and facilitate STAT/DNA interactions, which are intimately linked to conditional nuclear import or export signals. However, unlike other STATs, such as STAT1 and STAT2, which accumulate in the nucleus only following their phosphorylation, STAT3 can enter the nucleus independently of its phosphorylation. The mechanisms underlying these differences relate to the involvement of distinct importins used by STATs for their nuclear import. For instance, the phosphorylation of the nuclear localization signal of STAT1 is a prerequisite for its interaction with importin- $\alpha 5$ and subsequent nuclear import $[60,61]$. In contrast, STAT3 binds constitutively to importin- $\alpha 3$ and $\alpha 6$ [63], and the shuttling of STAT3 in and out of the nucleus seems independent of its phosphorylation [63].

\section{DNA binding of STATs without tyrosine phosphory- lation}

P-STAT1 binds to DNA in the form of homo- or heterodimers. Tyrosine phosphorylation is essential for the ligandinduced formation of STAT1 homodimers or STAT1-2 heterodimers and for their binding to the cis-recognition sequences in the promoters of IFN-regulated genes [13]. Without tyrosine phosphorylation, STAT1 does not regulate the transcription of IFN-inducible genes (e.g., IRF1) that have GAS sites in their promoters [37].

The high-resolution structures of DNA-bound STATs 1 and $3[64,65]$ and of the N-terminal portion of STAT4 [66] indicate that the mutual binding of two STAT monomers is possible without $\mathrm{SH} 2$-phosphotyrosine interactions. The structures indicate that each monomer of the DNA-bound STAT dimer contacts only half of the palindromic GAS element. Also, the N-terminal coiled coil domain of the dimer is likely to have very few contacts with DNA and thus is essentially free for additional protein-protein interactions $[64,66]$. Consistent with this, several recent studies have identified proteins that interact with this domain of STAT3 or other STATs [67-71]. U-STAT1 monomers can bind to DNA on their own by contacting one half of a palindromic GAS. They can also form dimers through mutual interaction of the N-terminal domain of each monomer and bind to GAS sequences in vitro [37]. The N-terminal region of STAT1, especially residues $135-200$ within the first coiledcoil domain, is required to form dimers of U-STAT1 on the LMP2 GAS in vitro. However, the affinity for U-STAT1 monomers or dimers is much less than that for P-STAT1 homodimers.

Weak STAT binding can be enhanced by increasing the concentration of STAT proteins or by interaction of STAT 1 with other proteins, either transcription factors bound to adjacent promoter sites or accessory factors and co-activators. In the case of the LMP2 gene, U-STAT1 binds to IRF1 through the N-terminal domain of STAT1, and the STAT1IRF1 dimer binds to the overlapping ICS2/GAS site on the LMP2 promoter [37]. The adenovirus E1A protein can down-regulate LMP2 transcription by interfering directly with this interaction of U-STAT1 and IRF1 and thus with their binding to the LMP2 promoter [37].

In addition to U-STAT1 [37], other U-STATs have been 
reported to be capable of binding to DNA. It has been shown that U-STAT6 associated with p300 binds directly to the COX-2 GAS element to drive its expression in NSCLC cells [10]; U-STAT3 competes with I $\mathrm{B}$ and binds to NFKB and, with the help of importin- $\alpha 3$, brings the USTAT3/p65/p50 complex to the nucleus, which then binds to a $\kappa B$ site to facilitate RANTES gene expression [9]. In addition to its interactions with NFאB, STAT3 has been shown to bind to other transcription factors. For example, it forms a complex with the CRE-binding protein on the JunB promoter [72] and with c-Jun on the $\alpha 2$-macrogloblin APRE [73]. Other reports show that STAT3 has an effect on CRE-like sites in the C/EBP $\beta$ promoter [74] and on the glucocorticoid response element [75], which lack classical GAS sequences. It should be mentioned that fewer than half of the genes that respond to high-level expression of U-STAT3 respond also to TNF- $\alpha$. The U-STAT3-responsive genes that do not respond to TNF- $\alpha$ probably do not have functional $\kappa \mathrm{B}$ elements, and two such genes do not need p65 in order to respond to U-STAT3. Therefore, it is extremely likely that U-STAT3 interacts productively with one or more transcription factors different from $\mathrm{NF \kappa B}$ to drive the expression of this class of genes. Identification of these factors and characterization of their interactions with U-STAT3 are of interest for future studies.

\section{The biology of U-STATs}

All seven STAT proteins share a high degree of sequence homology, but they are expressed differently. In resting cells, STATs reside largely in the cytoplasm as inactive homodimers [76]. However, upon ligand binding, receptor-associated JAKs become activated, leading to the phosphorylation of specific receptor tyrosine residues, which then direct the SH2-dependent recruitment of specific STATs, which in turn become JAK substrates. As activated STATs are released from the receptor they reorient into an antiparallel dimer, where the SH2 domain of one STAT binds to the phosphotyrosine of the other STAT. Activated STAT dimers translocate to the nucleus and bind to specific elements. STAT homodimers bind to members of the GAS family of enhancers (a palindrome, TTCNNNGAA). Different STATs are activated by phosphorylation of the tyrosine residue in response to different stimuli, for example, STAT 1 and 2 by type I and II IFNs, STAT 3 by IL- 6 and EGF, STATs 4 and 6 by IL-12 and IL-4, and STAT 5 by prolactin and IL-3, respectively [77-79]. As noted above, both the STAT1 and STAT3 genes are regulated by their own activation because their promoters contain GAS elements [80-82]. Long-term exposure of cells to IFNs leads to a large (20-fold or more) increase in the concentration of U-STAT1. Similarly, the STAT3 gene has a GAS element that drives its expression in response to the activation of STAT3, for example, in response to IL-6 [9, 82]. Hu et al. showed that priming with low concentrations of IFN- $\gamma$ for 2 days leads to increased tyrosine phosphorylation of STAT1 and increases total STAT1 expression in primary human monocytes in response to IFN- $\alpha$ [80]. Furthermore, the IL-6 and IFN- $\gamma$ receptors preferentially activate STAT3 and STAT1, respectively, but also activate the other STATs with lower efficiency. Qing et al. [81] showed that, when STAT1 is absent, IFN- $\gamma$ activates STAT3 robustly and, conversely, when STAT3 is absent, IL-6 and other gp130-linked cytokines activate STAT1 robustly [83]. It is interesting that, in addition to JAK1 and JAK2, SRC-family kinases are required to activate STAT3 in response to IFN- $\gamma[81]$. Because STAT1 and STAT3 usually have opposite biological effects, their reciprocal activation in response to IFN- $\gamma$ or IL-6 suggests that their relative abundance, which may vary considerably in different normal cell types, under different conditions, or in tumors, may well have a major impact on how cells behave in response to these two different classes of cytokines [81]. The ratio of STAT3 to STAT1 may be as much as hundreds of times different in cells pretreated with IL-6 than in cells pretreated with IFN- $\gamma$, with significant consequences for downstream signaling in response to a second cytokine that activates either STAT, for example, IFN $\beta$. Perhaps a more important consequence of up-regulated STAT expression is the ability of U-STATs 1 and 3 to drive gene expression through mechanisms distinct from those used by p-STATs. In recent experiments, van BoxelDezaire et al. have observed that, in freshly drawn human blood treated for 30-45 min with IFN- $\beta$, STATs 1,3 , and 5 are activated to very different extents in CD4+ and CD8+ $\mathrm{T}$ cells, B cells, and monocytes, with little activation of STAT1 in B cells (A van Boxel-Dezaire and GR Stark, unpublished observations). It seems inevitable, therefore, that the IFN-induced patterns of gene expression will be very different in these different cell types, as will the biological consequences of exposure to IFN.

The full biological relevance of the ability of P-STAT3 to increase the intracellular concentration of U-STAT3 remains to be established. In the context of cancer, the constitutive tyrosine phosphorylation of STAT3 in many different tumors is likely to lead to increased expression of U-STAT3, which in turn drives the expression of oncogenes such as MET and MRAS [9, 44]. In cell culture systems, long-term treatment with IL-6 increases total U-STAT3, and the levels of RANTES, MET, MRAS, and TIS11D [9, 44] are increased coordinately with U-STAT3. The biological role of U-STAT3-driven gene expression in normal physiology is best addressed by experiments with genetically altered mice. An important attempt to do this was reported by Narimatsu et al. [82], who mutated a GAS element of 
the endogenous STAT3 promoter. The ability of IL-6 to increase STAT3 expression was abrogated in some tissues but not in others, probably because STAT3-dependent expression of the STAT3 gene can be regulated through additional elements that were not recognized and therefore were not mutated. Incomplete suppression of the response of the STAT3 gene to IL-6 might well account for the mild phenotype of the promoter knock-in mouse observed by Naramatsu et al. Since complete deletion of STAT3 is embryonic lethal [84], it remains to be seen whether mice with complete loss of the STAT3-dependent induction of U-STAT3 expression would have severe defects, as might be expected if the up-regulation of U-STAT3 is important for the full physiological functions of the many cytokines that use the common gp130 receptor subunit to induce the phosphorylation of STAT3.

\section{Concluding remarks}

STATs are ancient transcription factors, present in Dictyostelium [85], Drosophila [86], and zebrafish [87]. The seven members of the mammalian STAT family range in size from 750 to 900 amino acids and feature several conserved domains, especially an SH2 domain. It is tempting to speculate that a primordial STAT first functioned as a constitutive transcription factor, with the ability to dimerize through ligand-dependent tyrosine phosphorylation as an additional function that was acquired more recently. Experiments in which the wild-type mouse genes encoding STAT1 or STAT3 are replaced cleanly by the Y-F or S-A mutants should give important new information to distinguish between the ligand-dependent and ligand-independent functions of these STATs in mammals.

\section{References}

1 Fu XY, Schindler C, Improta T, et al. The proteins of ISGF-3, the interferon alpha-induced transcriptional activator, define a gene family involved in signal transduction. Proc Natl Acad Sci USA 1992; 89:7840-7843.

2 Schindler $\mathrm{C}, \mathrm{Fu} \mathrm{XY}$, Improta $\mathrm{T}$, et al. Proteins of transcription factor ISGF-3: one gene encodes the 91-and 84-kDa ISGF-3 proteins that are activated by interferon alpha. Proc Natl Acad Sci USA 1992; 89:7836-7839.

3 Schindler C, Shuai K, Prezioso VR, et al. Interferon-dependent tyrosine phosphorylation of a latent cytoplasmic transcription factor. Science 1992; 257:809-813.

4 Darnell JE Jr. STATs and gene regulation. Science 1997; 277:1630-1635.

5 Levy DE, Darnell Jr JE. STATs: transcriptional control and biological impact. Nat Rev Mol Cell Biol 2002; 3:651-662.

6 Bowman T, Garcia R, Turkson J, Jove R. STATs in oncogenesis. Oncogene 2000; 19:2474-2488.

7 Bromberg JF. Activation of STAT proteins and growth control. Bioessays 2001; 23:161-169.
8 Chatterjee-Kishore M, Kishore R, Hicklin DJ, Marincola FM, Ferrone S. Different requirements for signal transducer and activator of transcription 1alpha and interferon regulatory factor 1 in the regulation of low molecular mass polypeptide 2 and transporter associated with antigen processing 1 gene expression. J Biol Chem 1998; 273:16177-16183.

9 Yang J, Liao X, Agarwal MK, et al. Unphosphorylated STAT3 accumulates in response to IL- 6 and activates transcription by binding to NFkappaB. Genes Dev 2007; 21:1396-1408.

10 Cui X, Zhang L, Luo J, et al. Unphosphorylated STAT6 contributes to constitutive cyclooxygenase- 2 expression in human non-small cell lung cancer. Oncogene 2007; 26:4253-4260.

11 Darnell JE Jr, Kerr IM, Stark GR. Jak-STAT pathways and transcriptional activation in response to IFNs and other extracellular signaling proteins. Science 1994; 264:1415-1421.

12 Ihle JN, Kerr IM. Jaks and Stats in signaling by the cytokine receptor superfamily. Trends Genet 1995; 11:69-74.

13 Stark GR, Kerr IM, Williams BR, Silverman RH, Schreiber RD. How cells respond to interferons. Annu Rev Biochem 1998; 67:227-264.

14 Durbin JE, Fernandez-Sesma A, Lee CK, et al. Type I IFN modulates innate and specific antiviral immunity. $J$ Immunol 2000; 164:4220-4228.

15 Muller M, Laxton C, Briscoe J, et al. Complementation of a mutant cell line: central role of the $91 \mathrm{kDa}$ polypeptide of ISGF3 in the interferon-alpha and -gamma signal transduction pathways. EMBO J 1993; 12:4221-4228.

16 Shuai K, Ziemiecki A, Wilks AF, et al. Polypeptide signalling to the nucleus through tyrosine phosphorylation of Jak and Stat proteins. Nature 1993; 366:580-583.

17 Bromberg JF, Horvath CM, Wen Z, Schreiber RD, Darnell JE Jr. Transcriptionally active Stat 1 is required for the antiproliferative effects of both interferon alpha and interferon gamma. Proc Natl Acad Sci USA 1996; 93:7673-7678.

18 Levy DE, Gilliland DG. Divergent roles of STAT1 and STAT5 in malignancy as revealed by gene disruptions in mice. Oncogene 2000; 19:2505-2510.

19 Boudny V, Kocak I, Lauerova L, Kovarik J. Interferon inducibility of STAT1 activation and its prognostic significance in melanoma patients. Folia Biol (Praha) 2003; 49:142-146.

20 Kovarik J, Boudny V, Kocak I, et al. Malignant melanoma associates with deficient IFN-induced STAT 1 phosphorylation. Int J Mol Med 2003; 12:335-340.

21 Kovarik P, Mangold M, Ramsauer K, et al. Specificity of signaling by STAT1 depends on SH2 and C-terminal domains that regulate Ser727 phosphorylation, differentially affecting specific target gene expression. EMBO J 2001; 20:91-100.

22 Dunn GP, Koebel CM, Schreiber RD. Interferons, immunity and cancer immunoediting. Nat Rev Immunol 2006; 6:836-848.

23 Kaplan DH, Shankaran V, Dighe AS, et al. Demonstration of an interferon gamma-dependent tumor surveillance system in immunocompetent mice. Proc Natl Acad Sci USA 1998; 95:75567561.

24 Lee CK, Rao DT, Gertner R, et al. Distinct requirements for IFNs and STAT1 in NK cell function. J Immunol 2000; 165:35713577.

25 Durbin JE, Hackenmiller R, Simon MC, Levy DE. Targeted disruption of the mouse Stat 1 gene results in compromised innate immunity to viral disease. Cell 1996; 84:443-450. 
26 Shankaran V, Ikeda H, Bruce AT, et al. IFNgamma and lymphocytes prevent primary tumour development and shape tumour immunogenicity. Nature 2001; 410:1107-1111.

27 Ikeda H, Old LJ, Schreiber RD. The roles of IFN gamma in protection against tumor development and cancer immunoediting. Cytokine Growth Factor Rev 2002; 13:95-109.

28 Wong LH, Krauer KG, Hatzinisiriou I, et al. Interferon-resistant human melanoma cells are deficient in ISGF3 components, STAT1, STAT2, and p48-ISGF3gamma. J Biol Chem 1997; 272:28779-28785.

29 Pansky A, Hildebrand P, Fasler-Kan E, et al. Defective Jak-STAT signal transduction pathway in melanoma cells resistant to growth inhibition by interferon-alpha. Int J Cancer 2000; 85:720-725.

30 Widschwendter A, Tonko-Geymayer S, Welte T, et al. Prognostic significance of signal transducer and activator of transcription 1 activation in breast cancer. Clin Cancer Res 2002; 8:30653074 .

31 Boudny V, Dusek L, Adamkova L, et al. Lack of STAT1 phosphorylation at TYR701 by IFNgamma correlates with disease outcome in melanoma patients. Neoplasma 2005; 52:330-337.

32 Timofeeva OA, Plisov S, Evseev AA, et al. Serine-phosphorylated STAT1 is a prosurvival factor in Wilms' tumor pathogenesis. Oncogene 2006; 25:7555-7564.

33 Kovacic B, Stoiber D, Moriggl R, et al. STAT1 acts as a tumor promoter for leukemia development. Cancer Cell 2006; 10:7787.

34 Kumar A, Commane M, Flickinger TW, et al. Defective TNF-alpha-induced apoptosis in STAT1-null cells due to low constitutive levels of caspases. Science 1997; 278:1630-1632.

35 Min W, Pober JS, Johnson DR. Kinetically coordinated induction of TAP1 and HLA class I by IFN-gamma: the rapid induction of TAP1 by IFN-gamma is mediated by Stat1 alpha. J Immunol 1996; 156:3174-3183.

36 White LC, Wright KL, Felix NJ, et al. Regulation of LMP2 and TAP1 genes by IRF-1 explains the paucity of CD8+ T cells in IRF-1 ${ }^{-/-}$mice. Immunity 1996; 5:365-376.

37 Chatterjee-Kishore M, Wright KL, Ting JP, Stark GR. How STAT1 mediates constitutive gene expression: a complex of unphosphorylated STAT1 and IRF1 supports transcription of the LMP2 gene. EMBO J 2000; 19: 4111-4122.

38 Lee CK, Gimeno R, Levy DE. Differential regulation of constitutive major histocompatibility complex class I expression in T and B lymphocytes. J Exp Med 1999; 190:1451-1464.

39 Stephanou A, Scarabelli TM, Brar BK, et al. Induction of apoptosis and Fas receptor/Fas ligand expression by ischemia/reperfusion in cardiac myocytes requires serine 727 of the STAT-1 transcription factor but not tyrosine 701. J Biol Chem 2001; 276:28340-28347.

40 Agrawal S, Agarwal ML, Chatterjee-Kishore M, Stark GR, Chisolm GM. Stat1-dependent, p53-independent expression of p21(waf1) modulates oxysterol-induced apoptosis. Mol Cell Biol 2002; 22:1981-1992.

41 Zhu X, Wen Z, Xu LZ, Darnell JE Jr. Stat1 serine phosphorylation occurs independently of tyrosine phosphorylation and requires an activated Jak2 kinase. Mol Cell Biol 1997; 17:6618-6623.

42 Nguyen H, Chatterjee-Kishore M, Jiang Z, et al. IRAK-dependent phosphorylation of Stat1 on serine 727 in response to interleukin-1 and effects on gene expression. J Interferon Cytokine Res 2003; 23:183-192.
43 Chatterjee-Kishore M, van den Akker F, Stark GR. Adenovirus E1A down-regulates LMP2 transcription by interfering with the binding of statl to IRF1. J Biol Chem 2000; 275:20406-20411.

44 Yang J, Chatterjee-Kishore M, Staugaitis SM, et al. Novel roles of unphosphorylated STAT3 in oncogenesis and transcriptional regulation. Cancer Res 2005; 65:939-947.

45 Shen X, Hong F, Nguyen VA, Gao B. IL-10 attenuates IFNalpha-activated STAT1 in the liver: involvement of SOCS2 and SOCS3. FEBS Lett 2000; 480:132-136.

46 Kiuchi N, Nakajima K, Ichiba M, et al. STAT3 is required for the gp 130-mediated full activation of the c-myc gene. J Exp Med 1999; 189:63-73.

47 Yang E, Lerner LR, Besser D, Darnell JE Jr. Independent and cooperative activation of chromosomal c-fos promoter by STAT3. J Biol Chem 2003; 278:15794-15799.

48 Jenab S. Quinones-Jenab V. The effects of interleukin-6, leukemia inhibitory factor and interferon-gamma on STAT DNA binding and c-fos mRNA levels in cortical astrocytes and C6 glioma cells. Neuroendocrinol Lett 2002; 23:325-328.

49 Senga T, Iwamoto T, Humphrey SE, et al. Stat3-dependent induction of BATF in M1 mouse myeloid leukemia cells. Oncogene 2002; 21:8186-8191.

50 Niu G, Bowman T, Huang M, et al. Roles of activated Src and Stat3 signaling in melanoma tumor cell growth. Oncogene 2002; 21:7001-7010.

51 Battle TE, Frank DA. The role of STATs in apoptosis. Curr Mol Med 2002; 2:381-392.

52 Hagihara K, Nishikawa T, Sugamata Y, et al. Essential role of STAT3 in cytokine-driven NF-kappaB-mediated serum amyloid A gene expression. Genes Cells 2005; 10:1051-1063.

$53 \mathrm{Yu}$ Z, Zhang W, Kone BC. Signal transducers and activators of transcription 3 (STAT3) inhibits transcription of the inducible nitric oxide synthase gene by interacting with nuclear factor kappaB. Biochem J 2002; 367:97-105.

54 Yoshida Y, Kumar A, Koyama Y, et al. Interleukin 1 activates STAT3/nuclear factor-kappaB cross-talk via a unique TRAF6and p65-dependent mechanism. J Biol Chem 2004; 279:17681776 .

55 Wen Z, Darnell JE Jr. Mapping of Stat3 serine phosphorylation to a single residue (727) and evidence that serine phosphorylation has no influence on DNA binding of Stat1 and Stat3. Nucleic Acids Res 1997; 25:2062-2067.

56 Kaptein A, Paillard V, Saunders M. Dominant negative stat3 mutant inhibits interleukin-6-induced Jak-STAT signal transduction. J Biol Chem 1996; 271:5961-5964.

57 Zhang X, Wrzeszczynska MH, Horvath CM, Darnell Jr JE. Interacting regions in Stat 3 and c-Jun that participate in cooperative transcriptional activation. Mol Cell Biol 1999; 19:7138-7146.

$58 \mathrm{Ng}$ YP, Cheung ZH, Ip NY. STAT3 as a downstream mediator of Trk signaling and functions. J Biol Chem 2006; 281:1563615644.

59 Meyer T, Begitt A, Lodige I, et al. Constitutive and IFN-gammainduced nuclear import of STAT1 proceed through independent pathways. EMBO J 2002; 21:344-354.

60 Sekimoto T, Nakajima K, Tachibana T, Hirano T, Yoneda Y. Interferon- $\gamma$-dependent nuclear import of Stat1 is mediated by the GTPase activity of Ran/TC4. J. Biol. Chem 1996; 271:3101731020 .

61 Sekimoto T, Imamoto N, Nakajima K, Hirano T, Yoneda Y. Ex- 
tracellular signal-dependent nuclear import of Stat 1 is mediated by nuclear pore-targeting complex formation with NPI-1, but not Rch1. EMBO J 1997; 16:7067-7077.

62 McBride KM, Banninger G, McDonald C, Reich NC. Regulated nuclear import of the STAT1 transcription factor by direct binding of importin- $\alpha$. EMBO J 2002; 21:1754-1763.

63 Liu L, McBride KM, Reich NC. STAT3 nuclear import is independent of tyrosine phosphorylation and mediated by importina3. Proc Natl Acad Sci USA 2005; 102:8150-8155.

64 Chen X, Vinkemeier U, Zhao Y, Jeruzalmi D, Darnell Jr JE, Kuriyan J. Crystal structure of a tyrosine phosphorylated STAT-1 dimer bound to DNA. Cell 1998; 93:827-839.

65 Becker S, Groner B, Muller CW. Three-dimensional structure of the Stat3beta homodimer bound to DNA. Nature 1998; 394:145151.

66 Vinkemeier U, Moarefi I, Darnell Jr JE, Kuriyan J. Structure of the amino-terminal protein interaction domain of STAT-4. Science 1998; 279:1048-1052.

67 Zhang JJ, Vinkemeier U, Gu W, et al. Two contact regions between Stat 1 and CBP/p300 in interferon gamma signaling. Proc Natl Acad Sci USA 1996; 93:15092-15096.

68 Zhu M, John S, Berg M, Leonard WJ. Functional association of Nmi with Stat5 and Stat1 in IL-2- and IFNgamma-mediated signaling. Cell 1999; 96:121-130.

69 Martinez-Moczygemba M, Gutch MJ, French DL, Reich NC. Distinct STAT structure promotes interaction of STAT2 with the $\mathrm{p} 48$ subunit of the interferon-alpha-stimulated transcription factor ISGF3. J Biol Chem 1997; 272:20070-20076.

70 Horvath CM, Stark GR, Kerr IM, Darnell Jr JE. Interactions between STAT and non-STAT proteins in the interferon-stimulated gene factor 3 transcription complex. Mol Cell Biol 1996; 16:6957-6964.

71 Chung CD, Liao J, Liu B, et al. Specific inhibition of Stat3 signal transduction by PIAS3. Science 1997; 278:1803-1805.

72 Kojima H, Nakajima K, Hirano T. IL-6-inducible complexes on an IL-6 response element of the junB promoter contain Stat3 and $36 \mathrm{kDa}$ CRE-like site binding protein(s). Oncogene 1996; 12:547-554.

73 Schaefer TS, Sanders LK, Nathans D. Cooperative transcriptional activity of Jun and Stat3 beta, a short form of Stat3. Proc Natl Acad Sci USA 1995; 92:9097-9101.

74 Niehof M, Streetz K, Rakemann T, et al. Interleukin-6-induced tethering of STAT3 to the LAP/C/EBPbeta promoter suggests a new mechanism of transcriptional regulation by STAT3. $J$ Biol Chem 2001; 276:9016-9027.

75 Zhang Z, Jones S, Hagood JS, Fuentes NL, Fuller GM. STAT3 acts as a co-activator of glucocorticoid receptor signaling. J Biol Chem 1997; 272:30607-30610.

76 Mertens C, Zhong M, Krishnaraj R, et al. Dephosphorylation of phosphotyrosine on STAT1 dimers requires extensive spatial reorientation of the monomers facilitated by the N-terminal domain. Genes Dev 2006; 20:3372-3381.

77 Hou J, Schindler U, Henzel WJ, et al. An interleukine-4-induced transcription factor: IL-4 Stat. Science 1994; 65:1701-1706.

78 Thierfelder WE, van Deursen JM, Yamamoto K, et al. Requirement for Stat4 in IL-12 mediated responses of natural killer and T-cells. Nature 1996; 382:171-174.

79 Takeda K, Akira S. STAT family of transcription factors in cytokine-mediated biological responses (Mini review). Cytokine Growth Factor Rev 2000; 11:199-207.

$80 \mathrm{Hu}$ X, Herrero C, Li WP, et al. Sensitization of IFN-gamma Jak-STAT signaling during macrophage activation. Nat Immunol 2002; 3:859-866.

81 Qing Y, Stark GR. Alternative activation of STAT1 and STAT3 in response to interferon-gamma. J Biol Chem 2004; 279:4167941685.

82 Narimatsu M, Maeda H, Itoh S, et al. Tissue-specific autoregulation of the stat 3 gene and its role in interleukin-6-induced survival signals in T cells. Mol Cell Biol 2001; 21:6615-6625.

83 Ramana CV, Gil MP, Schreiber RD, Stark GR. Stat1-dependent and independent pathways in IFN-gamma-dependent signaling. Trends Immunol 2002; 23:96-101.

84 Takeda K, Noguchi K, Shi W, et al. Targeted disruption of the mouse Stat3 gene leads to early embryonic lethality. Proc Natl Acad Sci USA 1997; 94:3801-3804.

85 Fukuzawa M, Araki T, Adrian I, Williams JG. Tyrosine phosphorylation-independent nuclear translocation of a dictyostelium STAT in response to DIF signaling. Mol Cell 2001; 7:779-788.

86 Hou SX, Zheng Z, Chen X, Perrimon N. The Jak/STAT pathway in model organisms: emerging roles in cell movement. Dev Cell 2002; 3:765-778.

87 Yamashita S, Miyagi C, Carmany-Rampey A, et al. Stat 3 controls cell movements during zebrafish gastrulation. Dev Cell 2002; 2: 363-375. 